\title{
COMMENT ON "THE COMPUTER AS THE ARTIST'S ALTER EgO"
}

Bernard Bel

Leonardo, vol. 23, no. 4. 1990, p. 461-462.

It is my pleasure to comment on Otto Laske's paper (Leonardo 23, No. 1, 53-66, 1990). I had the opportunity to read several published papers and preprints by Laske when preparing a one-day discussion on composition theory, which he recently was invited to chair at the Laboratoire Musique et Infonnatique de Marseille (MIM) •

As a computer scientist, I have been involved in research work with ethnomusicologists and contemporary musicians for a number of years [1]. Both the analysis of improvisational schemata of North Indian tabla players and the work with contemporary musicians have convinced me that looking at 'frozen' data (scores) yields a very narrow understanding of musical systems.

People (including musicians) who use computers are often stuck with the belief that the world beyond computer representations is very weakly related to the topic they are investigating. Taking just the opposite view, the romantic attitude stipulates that music (hence music making), like any 'high-level' human activity, cannot be captured in formal descriptions. Indeed, computer tools and methods available so far are still in infancy; but to ignore their potential role would be the denial of a positivist attitude toward progress, which I believe has always been a characteristic of art music (be it in western Europe or, say, in seventeenth-century Muslim India). A scientific attitude consists of trying to delineate musical reality as a whole [2] and then only making it clear which aspects of that reality are left out when it is reduced to formal descriptions - eventually, which formal aspects cannot be imbedded in (operative) computer implementations.

Epistemologists are concerned with the question of why we should at all need computers. The author makes it clear in the first paragraph that computers are useful in enabling artists "to distance themselves from conventional approaches". The statement is a counterpoint of the prevailing opinion that computers are no more than reliable storehouses of past experiences, to the extent that a memory-based paradigm of creativity is often put forward. As Stephen Smoliar observes, 'What Laske has overlooked is that the actual behaviour of making music cannot be abstracted away from the behaviour of listening to music and recalling what one has heard ... Ultimately, music is made so that one may listen to it .... The truth is that all the mind can do with music is compare it to past experiences" [ 31 .

What Smoliar has overlooked is the central role played by the axiologic approach in music, architecture and other design 
activities since the fifteenth century in Europe. We use the French word axiologie to designate "the larger set of a composer's compositional prerequisites: aesthetical, technical, philosophical, psychological, social, etc. In this way a composer's axiology is a larger factual field than his/her axiomatic, here taken to mean ... a set or a system of choices deliberately decided upon by the composer" [4].

Axiology is the immaterial aspect of what Laske calls 'task environment'. 'Subcognitive' activity, that is, mainly the memory of previous design tasks and the composer's 'inner listening', occurs at some stage in any of the three prototypical types of rule-based composition the author is describing. Although subcognitive activity is central to improvisational composition, it is also part of interpretative composition: organizing a randomly generated string of symbols requires some experience in dealing with abstract representations of music, which in turn means a long-term acquaintance with final products: sound and scores. Perhaps a relative weakness of the paper is that it is not explicit toward possible (and desirable) extensions of event-set generation beyond the serial music system. A point we discussed with the author is that an event list that is not generated on the basis of some theory of 'regularity' (e.g. serial music) may well be built on a descriptive base whose dimensions are qualitative or quantitative, imbedding topological time (a partial ordering of musical features). Resemblance/ dissemblance (multivalued) functions may then be computed, functions used for summarizing a structural description of the event universe as a Galois lattice [51. Resemblance is an alternative to truth assignment in musical 'semantics'. Any structure may then be viewed as transformations that are reflected as paths on the lattice.

I am not surprised that Laske's approach relates somehow to a method used for designing technical objects. Although traditional artists are not victims of the formalist folklore (the undesirable side effect of new approaches in Western culture), I have witnessed (although rarely) Indian musicians and dancers building a musical work or a ballet in a very similar, systematic way. Raja Chatrapati Singh's spoken introduction to the axiomatics of his 1985 drum solo composition Indira tal was almost as long as the performance itself. Therefore, I think there is some common sense grounding in the rule-based design method. If not, we would not be able to appreciate 'products' like the cathedral of Florence, classical Western music and the Eiffel Tower.

In conclusion, Laske is concerned with epistemology and aesthetics, but he is among the few who are attempting to drag them out of the realm of metaphysical speculations in order to gain insights into the essence of musical activity in its environment. Indeed, the computer-based environment described in his paper is a typical one in which intermediate representations of music (scores, whatever they may look like) play a central 
role. In the MIM laboratory composers use the real-time digital synthesizer SYTER along with acoustic instruments ('interaction' is taken to mean either sound generation or real-time sound sampling and processing, or both). This kind of set-up reintroduces the concept of a 'musical gesture' and the need for acquiring such gestures, in other words, the need for learning how to play the instrument designed. Perhaps neural nets would be good learning assistants. But this environment does not invalidate the requirement of a conceptual approach to musical structures.

Bernard Bel

Groupe Representation et Traitement des Connaissances (GRTC) Centre National de la Recherche Scientifique (CNRS)

31 , ch.J. Aiguier

13402 Marseille Cedex 09

France

\section{References}

1. Bernard Bel, "Time and Musical Structures", Interface, forthcoming; Jim Kippen and Bernard Bel, "Can the Computer Help Resolve the Problem of Ethnographic Description?"

Anthropological Quarterly 62, No. 3, 131-144 (1989); and Jim Kippen and Bernard Bel, "The Identification and Modelling of Percussion 'Language', and the Emergence of Musical Concepts in a Machine-Learning Experimental Set-Up", Computers and the Humanities 23, No. 3, 199-214 (1989).

2. Stephen W. Smoliar, 'Composition Theory and Memory', paper presented at the second conference of Musical Structures and Information Technology, Marseille, France, 1990 (pre-print).

3. Bernard Vecchione, "La Réalité musicale : Eléments d'épistémologie musicologique", thèse de Doctorat d'Etat, Université Paris VIII, 1985.

4. Bernard Vecchione, "Epistemologie, théorie et méthodologie de la composition (I): Remarques préliminaires et schéma méthodologique d'ensemble". In Rapport 1989, Groupe "Ordre et Chaos", Laboratoire Musique et Informatique de Marseille (MIM), 1990 (preprint), pp. 19-26.

5. See Vecchione [3]. 